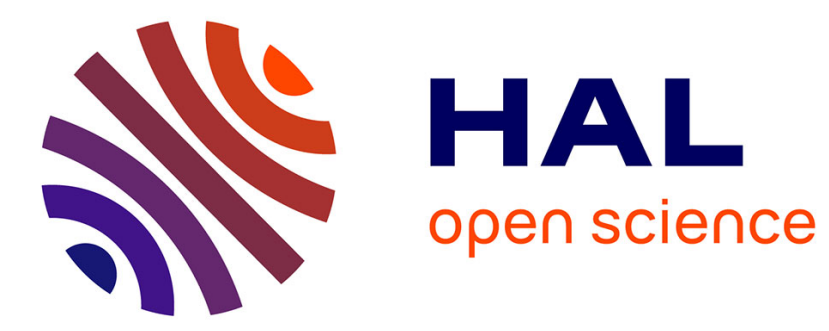

\title{
Core Network Function Placement in Mobile Networks
} Jad Oueis, Vania Conan, Damien Lavaux, Razvan Stanica, Fabrice Valois

\section{To cite this version:}

Jad Oueis, Vania Conan, Damien Lavaux, Razvan Stanica, Fabrice Valois. Core Network Function Placement in Mobile Networks. PIMRC 2017 - IEEE International Symposium on Personal, Indoor and Mobile Radio Communications, Oct 2017, Montreal, Canada. pp.5. hal-01573380

\section{HAL Id: hal-01573380 \\ https://hal.science/hal-01573380}

Submitted on 9 Aug 2017

HAL is a multi-disciplinary open access archive for the deposit and dissemination of scientific research documents, whether they are published or not. The documents may come from teaching and research institutions in France or abroad, or from public or private research centers.
L'archive ouverte pluridisciplinaire HAL, est destinée au dépôt et à la diffusion de documents scientifiques de niveau recherche, publiés ou non, émanant des établissements d'enseignement et de recherche français ou étrangers, des laboratoires publics ou privés. 


\title{
Core Network Function Placement in Mobile Networks
}

\author{
Jad Oueis, Razvan Stanica, Fabrice Valois \\ Vania Conan, Damien Lavaux \\ Univ Lyon, INSA Lyon, Inria, CITI, F-69621 Villeurbanne, France Thales Communications \& Security, France \\ Email: \{name.surname $\}$ insa-lyon.fr \\ Email: \{name.surname\}@thalesgroup.com
}

\begin{abstract}
An isolated base station is a base station having no connection to a traditional core network. To provide services to users, an isolated base station is colocated with an entity providing the same functionalities as the traditional core network, referred to as Local EPC. In order to cover wider areas, several base stations are interconnected, forming a network that should be served by a single Local EPC. In this work, we tackle the Local EPC placement problem in the network, to determine with which of the base stations the Local EPC must be co-located. We propose a novel centrality metric, flow centrality, which measures the capacity of a node to receive the total amount of flows in the network. We show that co-locating the Local EPC with the base station having the maximum flow centrality maximizes the total amount of traffic the Local EPC can receive from all base stations, under certain capacity and load distribution constraints. We compare the flow centrality to other state of the art centrality metrics, and emphasize its advantages.
\end{abstract}

\section{INTRODUCTION}

Mobile networks have always relied on a fixed, hierarchical, and standardized architecture. In the LTE architecture, the Evolved Packet Core (EPC) is an all-IP core network that provides the user with IP connectivity to packet data networks, and manages the network [1]. The EPC and the access network are connected via backhaul links, cautiously dimensioned to support peak data rates and high-speed services.

Recently, the concept of isolated base stations (BSs) disrupted this traditional network architecture. A BS is referred to as isolated when it has no backhaul connectivity to a traditional core network [2]. Isolated BSs are innovative solutions providing users with at least local data services, when a classical network fails or does not exist [3]. Use cases include out-of-coverage situations where no network infrastructure exists, failures due to increased network demand, and network infrastructure destruction following man-made or natural disasters [4].

In order to provide services without backhaul communication, an isolated BS must have access to a Local EPC. A Local EPC is an entity analogous to the traditional EPC, providing the same basic functionalities as the latter. Unlike a traditional EPC, the Local EPC is co-located with the BS [2]. In order to cover wider areas, several isolated BSs must be deployed, forming a network of interconnected BSs served by a single Local
EPC. All those BSs must be able to reach the designated Local EPC, co-located with one of them. Hence, the Local EPC placement problem arises, questioning where should the Local EPC be placed in order to better serve the network. In other words, with which of the BSs must the Local EPC be co-located [3].

In this work, we argue that a Local EPC should be placed in a way that allows it to receive the maximum possible traffic from the BSs in the network, under certain capacity and load distribution constraints. To that end, we propose a new centrality metric, flow centrality, which measures the capacity of a node in receiving the total amount of flows in the network. The flow centrality of a node is represented by the maximum uniform traffic that can be sent simultaneously by all the other nodes in the network towards this node, while respecting link capacity constraints. Consequently, the Local EPC should be co-located with the node having the maximum flow centrality. Following a comparison with different centrality metrics, we show the loss in the total amount of traffic that can be received by the Local EPC, when the latter is placed on a node not having the maximum flow centrality.

The paper is organized as follows. In Sec. II, we discuss related works. In Sec. III, the network model is described. We introduce the flow centrality metric in Sec. IV, and analyze its properties in Sec. V. In Sec. VI, we compare the flow centrality metric to other relevant centrality metrics. Finally, Sec. VII concludes the paper.

\section{RELATED WORK}

Isolated BSs recently appeared in the standardization of the Isolated E-UTRAN Operation for Public Safety (IOPS) feature by the 3rd Generation Partnership Program (3GPP). IOPS aims to ensure continued service to public safety users via isolated BSs [2]. In this sense, Gomez et al. [5] proposed a software architecture with a set of protocols allowing the virtualization of the EPC functions in order to co-locate them with the BS. Our contribution is complementary to these works, since we determine where to place the Local EPC serving a network with multiple isolated BSs.

The placement problem appears in literature under different forms, such as the gateway placement prob- 
lem in wireless mesh networks (WMN). As opposed to our work, where only the placement of one Local EPC is needed in the network, several gateways can be placed in a WMN, with the objective of optimizing their placement, under different quality of service constraints, such as bandwidth requirements [6]-[8], and delay constraints [6]. Placing virtualized network functions (VNF) is another similar problem. VNF placement is usually done on a per-flow basis, with the VNF as a middle point between a source and a destination [9]. Conversely, we consider the overall traffic of a source node, encompassing all of the flows, with the Local EPC as an endpoint.

Our intuition is that the Local EPC should be placed on a "central" node. There are several ways to measure node centrality in a network, each one highlighting a different node characteristic [10]. The degree centrality of a node is equal to the number of links the node has with other nodes [11]. The degree centrality gives an idea on the node connectivity in the network, but does not take into consideration the limited link capacities. The weighted degree is equal to the sum of the weights of the links connecting the node to its direct neighbors [11]. While a node with the maximum weighted degree is potentially capable of receiving the maximum amount of traffic, this traffic is not necessarily achievable, due to other limited links. The closeness centrality of a node measures how close the node is to all the other nodes in the network [12]. The position of a node with the maximum closeness centrality gives it a relative advantage for easily communicating with all the other nodes. However, the limited link capacities leading to this central node could limit the amount of traffic it is capable of receiving. The betweenness centrality of a node quantifies the number of times a node falls on the shortest path between two other nodes [13]. The node with the maximum betweenness centrality is well placed for forwarding communications between the nodes. However, link capacities around that node may limit its ability to forward traffic. In this paper, we further highlight the inadequacy of these centrality measures for the Local EPC placement. We show how placing the Local EPC on a node maximizing one of these centralities reduces the amount of traffic the Local EPC is capable of receiving.

\section{NETWORK MODEL}

We consider a network of interconnected isolated BSs, that have no backhaul connectivity to a traditional EPC. All these BSs must access a Local EPC in order to be able to serve users. A single Local EPC serves the network, and is co-located with one of the BSs. A Local EPC provides the same functionalities as the traditional EPC, supports bearer services, and hosts the application servers. As the local EPC is co-located with one of the BSs, the inter-BS links will be responsible of forwarding all data and signaling traffic between each BS and the
Local EPC, respectively. Exchanged traffic on the links between a BS and the Local EPC is routed either directly, if the Local EPC is at one hop from the BS, or through interconnected BSs in a multi-hop fashion.

The design of inter-BS links is out of the scope of this paper. Regardless of the wireless technology used, we consider that there is no contention between those links for resource utilization, and their bandwidth is limited. We assume that interfering wireless links are operating on distinct channels, allowing parallel transmissions on the different links, with no interference [6].

Let $G(V, E)$ be an undirected graph modeling our network, with $|V|$ nodes, and $|E|$ edges. Each BS is a node of the graph, and the inter-BS links are the graph edges. The BS co-located with the Local EPC serving the network, denoted by $d$, is considered as the destination node in the graph, while all the other BSs in the network are sources. Let $\mathcal{S}=V \backslash\{d\}$, and $\mathcal{D}=\{d\}$ be the set of sources and destinations of $G$, respectively. To model the inter-BS links with limited bandwidth, we consider graph edges with limited capacities, where $c(u, v)$ is the capacity of an edge $(u, v) \in E$, and $f(u, v)$ the flow through this edge. To model the traffic between a BS and the Local EPC, we denote by $z(v, d)$ the flow that a source node $v$ sends towards the destination node $d$.

\section{Flow Centrality}

\section{A. Local EPC Placement Criteria}

The Local EPC must be able to receive (transmit) all the data and signaling traffic generated by (destined to) the BS. Hence, the local EPC placement depends on the amount of traffic routed in the network, which in turn depends on the number of users, and their requests. Traffic is aggregated and forwarded by intermediate BSs towards the Local EPC. The load of the congested interBS links is upper bounded by their respective limited capacities, creating a bottleneck. Thus, the Local EPC placement must take into consideration the capacity of the inter-BS links, to ensure that all traffic can circulate in the network without losses. On the other hand, the number of users and their requests are dynamic. Hence, the optimal position of the Local EPC might change depending on the traffic of each BS. While such information may not be available at early deployment, we propose to place the Local EPC in a way allowing us to cover the largest number of scenarios. Therefore, we treat all BSs as equals, and uniformly maximize the amount of traffic they can send. We suppose that each BS in the network can send an amount of traffic $\lambda(d)$ towards the Local EPC co-located with node $d$, and we recommend placing the Local EPC in such a way that $\lambda(d)$ is maximized. This maximizes the possible amount of traffic that all BSs are capable of forwarding to the Local EPC simultaneously, while respecting the limited link capacities. 


\section{B. Flow Centrality: A Metric For Local EPC Placement}

This placement criteria allows us to define flow centrality, a novel centrality metric measuring the capacity of a node in receiving the total amount of flows in the graph. The flow centrality of a node is represented by the maximum traffic that can be simultaneously generated by all the other nodes in the graph, and directed towards this node as unique destination. To compute the flow centrality of node $d$, it is sufficient to compute the maximum amount of flows that node $d$ can receive from all the other nodes in the graph. As each node $v \in V$ has a supply $z(v, d)=\lambda(d)$ to send towards $d$, then the total flow value received at node $d$ must be: $|f|_{d}=\sum_{v \in \mathcal{S}} z(v, d)=(n-1) \cdot \lambda(d)$

We denote by $\bar{\lambda}(d)$ the maximum achievable value of $\lambda(d)$. The value of $\bar{\lambda}(d)$ is obtained through the maximization problem of $\lambda(d)$ (Eq. 1), subject to the following constraints: all sources in the graph have a fixed and uniform supply equal to $\lambda(d)$ (Eq. 2); the flow on each edge in the graph must not surpass the edge capacity (Eq. 3); the flow entering a node must be equal to the flow exiting a node (Eq. 4); the total flow value received at the sink is equal to the sum of all the supplies of the sources (Eq. 5).

$$
\begin{aligned}
\underset{\lambda(d) \in[0, \infty)}{\operatorname{Maximize}} & \lambda(d) \\
\text { subject to } & z(v, d)=\lambda(d), \forall v \in \mathcal{S} \\
& f(u, v) \leq c(u, v), \forall(u, v) \in E \\
& \sum_{u \in \mathcal{S}} f(u, v)=\sum_{w \in \mathcal{S}} f(v, w), \forall v \in \mathcal{S} \\
& \sum_{v \in V} f(v, d)=(n-1) \cdot \lambda(d)
\end{aligned}
$$

When $\lambda(d)$ is maximized, the total flow received at $d$ is maximum, such that: $|f|_{d_{\max }}=(n-1) \cdot \bar{\lambda}(d)$. Eventually, the flow centrality of a node $d$ is defined as $\bar{\lambda}(d)$. The maximum flow centrality $\lambda_{\max }$ is then expressed as: $\lambda_{\max }=\max _{d \in V}(\bar{\lambda}(d))$. Practically, in order to better serve the network, the Local EPC must be co-sited with the node $v$ having the maximum flow centrality, such that $\bar{\lambda}(v)=\lambda_{\max }$.

\section{Flow Centrality Properties}

We take in Fig. 1a an example of a network topology, with isolated BSs served by a Local EPC. The network is represented by a random geometric graph, where 10 nodes are randomly placed in space, and two nodes are connected by a link if and only if their distance is smaller than a certain radius $r$. Link capacities are randomly distributed such that $c \in[0,100]$ (units of traffic). We compute the flow centrality value of each node using the CPLEX software package. For this network with a relatively small number of nodes, the overall computation time is in the order of milliseconds. This small number of nodes corresponds to the nature of the networks we consider, where only a few BSs are needed. Nevertheless, it should be noted that, with the increase of the number of nodes, the computation time increases accordingly, but remains in the order of seconds even for networks with 100 nodes.

Fig. 1b shows the computed flow centrality value $\bar{\lambda}(d)$ of each node $d$. Results show that node 2 has the maximum flow centrality, hence $\lambda_{\max }=\bar{\lambda}(2)$.

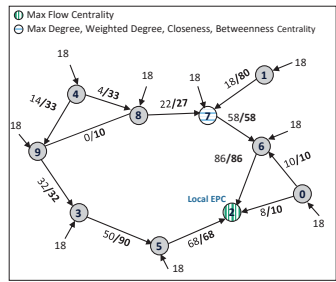

(a)

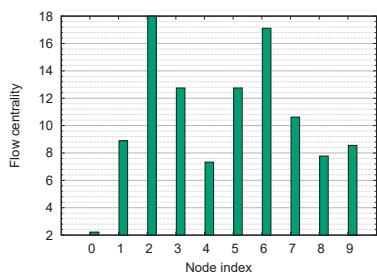

(b)
Fig. 1. (a) A random geometric graph topology. On each link $(u, v)$, we show $f(u, v) / c(u, v)$, i.e. the flow routed on the link in comparison with the maximum link capacity, if all nodes transmit $\lambda_{\max }=18$ units of traffic towards the Local EPC co-located with node 2; (b) The flow centrality values of the nodes of the graph in Fig.1a.

We show in Fig. 1a the values of the flows routed on each link, in comparison with the maximum link capacity, when the Local EPC is placed at node 2, and each node sends $\lambda_{\max }=18$ units of traffic towards the Local EPC. We notice that, while some links are saturated, others are under-used. Saturated links are the ones that upper bound the value of $\lambda_{\max }$. For this particular topology and this capacity distribution, those links are: $(9,3),(5,2)$, and $(7,6)$. Increasing (decreasing) the capacity of those links increases (decreases) the value of $\lambda_{\max }$. On the other hand, link $(1,7)$, which is only used to route traffic from node 1, is under-used. However, increasing the traffic sent by node 1 renders the total flow unfeasible, due to the saturated links leading to the destination node.

From these results, it is clear that the value $\bar{\lambda}(d)$ of a node $d$ is dictated by the capacity of the links leading to that node, as well as by the capacity of all the links in the graph. In the following, we consider a sample of 100 random geometric graphs of radius $r$, with random link capacities distribution such that $c \in\left[c_{\min }, c_{\max }\right]$. We denote by $c_{\text {avg }}$ the average link capacity, such that $c_{a v g}=\frac{c_{\min }+c_{\max }}{2}$, and by $\Delta c$ the capacity range, such that $\Delta c=c_{\max }-c_{\min }$.

To highlight the proportional relation between the maximum flow centrality $\lambda_{\max }$ and the link capacities, we show, in Fig. 2, the variation of the average value of $\lambda_{\max }$ function of the average capacity $c_{\text {avg }}$. By varying the values of $c_{\text {avg }}$ but keeping a constant interval $\Delta c$, we compute the average value of the maximum flow centrality $\lambda_{\max }$ obtained for each value of $c_{a v g}$. We show this variation for different values of the graph radius $r$. The higher $r$ is, the higher the number of links in the network. Results in Fig. 2 show that the average 
value of $\lambda_{\max }$ increases linearly with the increase of $c_{\text {avg. }}$. For $r=1$, where almost all pairs of nodes are connected, the value of $\lambda_{\max }$ is approximately equal to $c_{\text {avg }}$. This suggests that the value of the maximum flow centrality is upper bounded by the average link capacity. As we compare different values of capacity intervals, such as $\Delta c=5$ and $\Delta c=10$, we notice that the value of $\lambda_{\max }$ and its variation depending on $c_{a v g}$ are very similar in both cases. Thus, while the value of $\lambda_{\max }$ depends on the average link capacity, the capacity range is practically irrelevant. It should be noted, however, that the capacity range does have an impact on the position of the node with the maximum flow centrality.

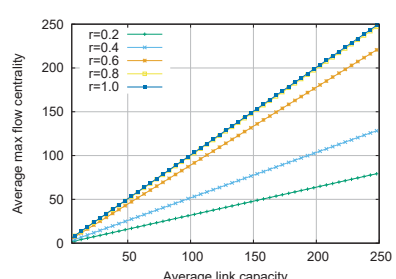

(a) $\Delta c=5$

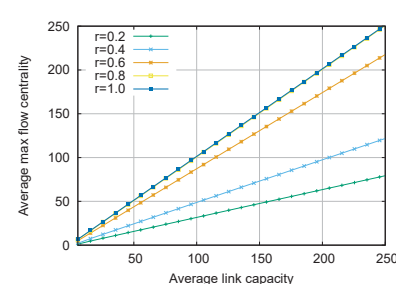

(b) $\Delta c=10$
Fig. 2. Variation of the average maximum flow centrality $\lambda_{\max }$ function of the average link capacity $c_{a v g}$, for a constant capacity range $\Delta c$, and different values of graph radius $r$.

\section{BENCHMARKING Flow CEnTRALity}

In this section, we compare the flow centrality with the centrality metrics, discussed in Sec. II, that intuitively appear to be the closest to it. We define the matching percentage as the percentage of scenarios where the node with maximum flow centrality is identical to the node maximizing one of the other centralities. To study whether one of the centrality metrics could be used for the Local EPC placement instead of the flow centrality, we define the relative traffic loss $\epsilon_{\lambda}(u)$ as the loss incurred if the Local EPC was placed on a node $u$ maximizing one of the centrality metrics, but not the flow centrality. If $\lambda_{\max }$ is the maximum flow centrality, and $\bar{\lambda}(u)$ is the flow centrality of node $u$, then:

$$
\epsilon_{\lambda}(u)=\frac{\lambda_{\max }-\bar{\lambda}(u)}{\lambda_{\max }}
$$

In the following, we study different network topologies. We fix the average link capacity $c_{a v g}$, and vary the capacity range $\Delta c$. All the results are averaged on samples of 100 randomly generated graphs, with confidence intervals of $95 \%$.

\section{A. Grid Graphs}

We first consider grid graphs with $n=x \times y$ nodes. We show examples of grids such that $n=\{2 \times 5,3 \times 3,3 \times 4\}$. We vary the capacity range $\Delta c$, such that $\Delta c=\{0,10,40,100\}$. Fig. 3 shows, for different grid dimensions, the matching percentage, i.e., the percentage of cases where the node with maximum flow centrality is identical to the node with the maximum closeness centrality, on one side, and to the node with the maximum weighted degree centrality, on the other. It is clear in Fig. 3 that, when link capacities are uniform, i.e., $\Delta c=0$, the matching percentage is $100 \%$. This means that the node with the maximum flow centrality is also the central node, which maximizes both closeness and weighted degree centralities. However, if links in the graph have different capacities, i.e., $\Delta c \neq 0$, then these nodes are not necessarily identical. The central node does not necessarily have the maximum weighted degree centrality, due to the random link capacities. On the other hand, having the maximum weighted degree centrality does not necessarily mean that the node is capable of receiving the maximum amount of traffic from all the other nodes. Moreover, we notice, in some cases, changes in the value of the matching percentage for different values of $\Delta c$. This is because $\Delta c$ affects the position of the node with the maximum flow centrality. These conclusions are rather consistent for the different grid dimensions.

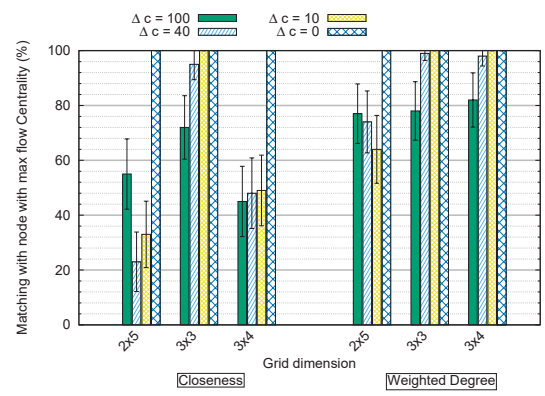

Fig. 3. The percentage of scenarios where the node with maximum flow centrality is identical to a node maximizing one of the other centralities, for grids of different dimensions, for different capacity ranges, and with constant average capacity.

We compute now the relative traffic loss $\epsilon_{\lambda}$ (Eq.6) if the Local EPC was placed on the node with the maximum closeness centrality or the maximum weighted degree centrality. Fig. 4 shows that the relative loss is important, with an average ranging between $35 \%$ and $55 \%$, for both centralities. Interestingly, even tough the node with the maximum weighted degree had higher matching percentages with the one maximizing flow centrality, the loss when the two nodes are different is slightly higher than the loss corresponding to the closeness centrality.

\section{B. Random Geometric Graphs}

We consider now random geometric graphs with 10 nodes, on a total surface of one unit square, and a radius $r=0.2$. We vary the link capacities such that $\Delta c=\{0,10,40,100\}$. We show in Fig. 5 the matching percentage between the node with maximum flow centrality and each of the other centralities. We notice that, unlike grid graphs, even with uniform link capacities, 


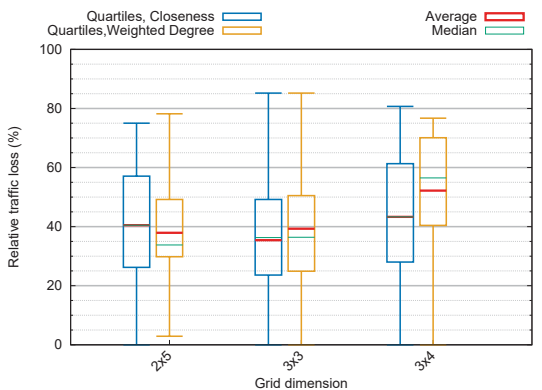

Fig. 4. Relative traffic loss when the Local EPC is placed on the node maximizing the closeness centrality and the weighted degree centrality, for grids of different dimensions, for $\Delta c=100$

i.e., $\Delta c=0$, the node with the maximum flow centrality can be different from the nodes that maximize the other centralities. On the other hand, when link capacities are randomly distributed, results in Fig. 5 show that, for all values of $\Delta c$, the closeness centrality is the closest to the flow centrality in terms of matching percentage. For example, for $\Delta c=100$, the node with maximum flow centrality matches the node with the maximum closeness centrality in $95 \%$ of the cases.

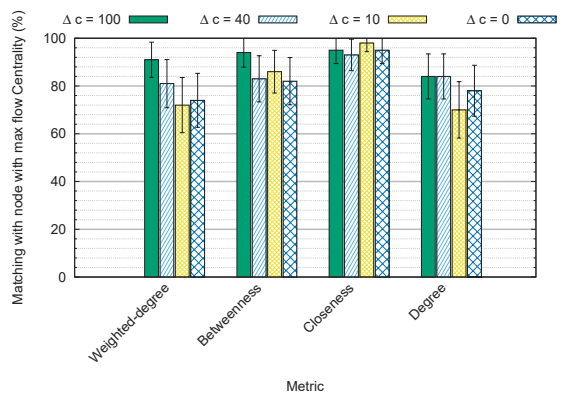

Fig. 5. The percentage of scenarios where the node with maximum flow centrality is identical to a node maximizing one of the other centralities, in random geometric graphs, for different capacity ranges, and with constant average capacity.

In Fig. 6, we show the relative traffic loss $\epsilon_{\lambda}$ (Eq.6) when the Local EPC is not placed on the node with the maximum flow centrality. Even though nodes with maximum closeness centrality have the highest matching percentage with the nodes with maximum flow centrality, results show that the average loss incurred when these nodes are different is relatively high. Fig. 6 indicates that placing the Local EPC on the node with the maximum closeness centrality instead of the node with the maximum flow centrality would cause an average loss of $46 \%$, the highest loss in comparison with the other centrality measures. The other relative losses are lower, but still important, around $30 \%$.

\section{CONCLUSION}

In conclusion, we tackled the placement problem of a Local EPC serving a network of isolated BSs with no backhaul connectivity. We proposed the flow centrality

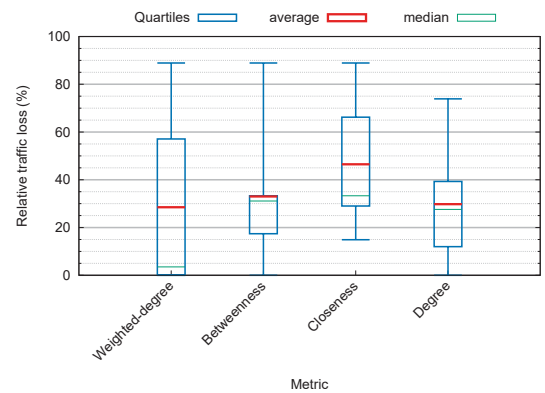

Fig. 6. Relative traffic loss when Local EPC is placed on the node maximizing centrality metrics other than the flow centrality, in random geometric graphs, for $\Delta c=100$.

metric, and showed that co-locating the Local EPC with the BS having the maximum flow centrality maximizes the total amount of traffic that the Local EPC is capable of receiving from all the BSs. Treating the BSs in the network equally, by uniformly maximizing their capabilities, is suitable for a network where the Local EPC placement is pre-planned. For future work, non-uniform BS demands must also be considered. Furthermore, endto-end delay constraints must be further included in the Local EPC placement criteria.

\section{REFERENCES}

[1] M. Sauter, "From GSM to LTE-advanced: An Introduction to Mobile Networks and Mobile Broadband", John Wiley \& Sons, 2014.

[2] 3GPP TR 23.797, "Technical Specification Group Services and System Aspects; Study on architecture enhancements to support isolated Evolved Universal Terrestrial Radio Access Network (EUTRAN) operation for public safety (Release 13)", June 2015.

[3] J. Oueis, V. Conan, D. Lavaux, R. Stanica, F. Valois, "Overview on LTE Isolated E-UTRAN Operation for Public Safety”, IEEE Communications Standards Magazine, 1(2), 98-105, July 2017.

[4] A. Valcarce, T. Rasheed, K. Gomez, S. Kandeepan, L. Reynaud, R. Hermenier, A. Munari, M. Mohorcic, M. Smolnikar, I. Bucaille, "Airborne Base Stations for Emergency and Temporary Events", Proc. PSATS, Toulouse, France, June 2013.

[5] K. Gomez, L. Goratti, T. Rasheed, L. Reynaud, "Enabling Disaster-Resilient 4G Mobile Communication Networks", IEEE Communications Magazine, 52(12), 66-73, Dec. 2014.

[6] B. Aoun, R. Boutaba, Y. Iraqi, G. Kenward, "Gateway Placement Optimization in Wireless Mesh Networks with QoS Constraints", IEEE Journal in Selected Areas of Communication, 24(11), 21272136, Nov. 2006

[7] L. Qiu, R. Chandra, K. Jain and M. Mahdian, "Optimizing the Placement of Integration Points in Multi-hop Wireless Networks", Proc. ICNP, Berlin, Germany, Oct. 2004.

[8] S. N. Muthaiah, C. Rosenberg, "Single Gateway Placement in Wireless Mesh Networks", Proc. ISCN, Istanbul, Turkey, June 2008.

[9] M. Bouet, J. Leguay, T. Combe, V. Conan, "Cost-based Placement of vDPI Functions in NFV Infrastructures", International Journal of Network Management, 25(6), 490-506, Nov. 2015.

[10] S. P. Borgatti, "Centrality and Network Flow", Social networks, 27(1), 55-71, Jan. 2015.

[11] L. C. Freeman, "Centrality in Social Networks: Conceptual Clarification", Social Networks, 1(3), 215-239, Jan. 1978.

[12] A. Bavelas, "Communication Patterns in Task Oriented Groups", The Journal of the Acoustical Society of America, 22(6), 271-282, Nov. 1950.

[13] L.C. Freeman, "A Set of Measures of Centrality Based on Betweenness", Sociometry, 35-41, 1977. 